\title{
La pasteurisation à l'aide de la méthode de Holder appliquée au lait maternel provenant de donneuses permet d'inactiver le SRAS-CoV-2
}

\author{
Sharon Unger MD, Natasha Christie-Holmes PhD, Furkan Guvenc HBSc, Patrick Budylowski HBSc, \\ Samira Mubareka MD, Scott D. Gray-Owen PhD, Deborah L. O'Connor PhD RD
}

— Citation : CMAJ 2020 August 4;192:E871-4. doi : 10.1503/cmaj.201309-f; diffusion hâtive le 9 juillet 2020

Voir la version anglaise de l'article ici : www.cmaj.ca/lookup/doi/10.1503/cmaj.201309

\section{RÉSUMÉ}

CONTEXTE : Le recours aux dons de lait maternel pasteurisé est la norme de soins dans les hôpitaux pour les nourrissons ayant un très faible poids à la naissance, afin de faire le pont en attendant que les mères puissent allaiter leur enfant. Le but de cette étude était de vérifier si la pasteurisation à l'aide de la méthode de Holder (à $62,5{ }^{\circ} \mathrm{C}$ pendant $30 \mathrm{~min}$ ) serait suffisante pour inactiver le coronavirus du syndrome respiratoire aigu sévère 2 (SRAS-CoV-2) dans des échantillons de lait maternel provenant de donneuses.

MÉTHODES : Nous avons inoculé avec le SRAS-CoV-2 des échantillons de lait congelés provenant de 10 donneuses de la Rogers Hixon Ontario Human Milk Bank (la banque de lait maternel de l'Ontario) pour atteindre une concentration finale de $1 \times 10^{7} \mathrm{DICT}_{50} / \mathrm{mL}$ (50\% de la dose infectante de la culture de tissus par $\mathrm{mL}$ ). Les échantillons ont été pasteurisés à l'aide de la méthode de Holder ou laissés à la température du laboratoire pendant 30 minutes, puis nous avons mis en culture des dilutions en série sur des cellules Vero E6 durant 5 jours. Nous avons utilisé des échantillons témoins dans cette étude, soit des échantillons de lait provenant des mêmes donneuses, auxquels le virus n'a pas été ajouté (échantillons pasteurisés et non pasteurisés), de même que des réplicats de cellules Vero E6 directement inoculées avec le SRAS-CoV-2. Nous rapportons ici les effets cytopathologiques en $\mathrm{DICT}_{50} / \mathrm{mL}$.

RÉSULTATS : Nous n'avons détecté aucune activité cytopathologique dans l'ensemble des échantillons de lait contenant le SRAS-CoV-2 pasteurisés à l'aide de la méthode de Holder. Dans les échantillons contenant le SRASCoV-2 qui n'ont pas été pasteurisés, mais plutôt laissés à la température du laboratoire pendant 30 minutes, nous avons observé une réduction du titre infectieux d'environ $1 \mathrm{log}$.

INTERPRÉTATION : La pasteurisation du lait maternel à l'aide de la méthode de Holder (à $62,5^{\circ} \mathrm{C}$ pendant $30 \mathrm{~min}$ ) inactive le SRAS-CoV-2. Ainsi, si du lait maternel provenant de donneuses contenait le virus à la suite d'une transmission par la glande mammaire ou d'une contamination, cette méthode de pasteurisation rendrait le lait sans danger pour la consommation par le nourrisson et la manipulation par les travailleurs de la santé.
$\mathbf{L}$

e lait maternel est la plus importante source de nutrition pour les nourrissons, et il contient une multitude de facteurs bioactifs et immunomodulateurs, dont les cytokines, la lactoferrine, les oligosaccharides et les immunoglobulines sécrétoires, qui contribuent au développement du système immunitaire et protègent contre les infections des voies respiratoires et du système gastrointestinal ${ }^{1-5}$. Chez les nourrissons vulnérables, comme ceux ayant un très faible poids à la naissance $(<1500 \mathrm{~g}$ à la naissance), la consommation de lait maternel est associée à un séjour réduit à l'hôpital et à une diminution du risque de septicémie et d'entérocolite nécrosante néonatale, un problème intestinal grave ${ }^{6-9}$. La norme de soins dans les hôpitaux du Canada recommande que l'on donne aux nourrissons ayant un très faible poids à la naissance du lait maternel pasteurisé provenant de banques de lait maternel jusqu'à ce que la mère produise suffisamment de lait ${ }^{10}$.

Dans le passé, les épidémies mondiales, comme celle du $\mathrm{VIH} /$ sida, ont eu des effets dévastateurs sur les banques de lait maternel en raison des risques perçus. Dans les années 1980, la recherche ayant montré que le VIH pouvait se transmettre par le lait maternel, 22 des 23 banques canadiennes de lait maternel ont fermé leurs portes ${ }^{11}$. Tout comme le VIH, plusieurs autres virus peuvent se transmettre par le lait maternel, comme le virus de l'hépatite, le cytomégalovirus et le virus 
T-lymphotrope 1 humain ${ }^{12}$. Certains virus peuvent être sécrétés dans le lait par passage paracellulaire, alors que les jonctions serrées se séparent en réaction à une maladie ou à une inflammation chez la mère ${ }^{3}$. Les autres modes de transmission sont notamment la contamination par les gouttelettes respiratoires, la peau, les tire-lait et les contenants de lait. Les banques de lait affiliées à la Human Milk Banking Association of North America (HMBANA) et à la European Milk Bank Association (EMBA) utilisent la méthode de Holder pour pasteuriser le lait (à $62,5^{\circ} \mathrm{C}$ pendant $30 \mathrm{~min}$ ) avant sa distribution; la méthode de Holder est efficace pour inactiver les virus précédemment mentionnés ${ }^{13,14}$.

Peu de données sont disponibles sur la prévalence du coronavirus du syndrome respiratoire aigu sévère 2 (SRAS-CoV-2) dans le lait maternel, ou sur son infectivité. Toutefois, le virus a été détecté dans le lait maternel par un test d'amplification en chaîne par polymérase couplée à une transcription inverse (RT$P C R)^{15-18}$. Les mères qui font des dons de lait dans les banques affiliées à la HMBANA sont soumises à un dépistage verbal de la maladie à coronavirus 2019 (COVID-19), mais le dépistage direct du SRAS-CoV-2 par écouvillonnage du nasopharynx et RT-PCR n'est pas requis. Bien qu'il n'y ait pas de preuves directes montrant que la pasteurisation à l'aide de la méthode de Holder puisse inactiver le SRAS-CoV-2 dans le lait maternel, on sait que ce virus est sensible à la chaleur ${ }^{19}$. Le but de cette étude était de confirmer que la pasteurisation à l'aide de la méthode de Holder est suffisante pour inactiver le SRAS-CoV-2 dans des échantillons de lait maternel provenant de donneuses.

\section{Méthodes}

\section{Modèle d'étude}

La Rogers Hixon Ontario Human Milk Bank de Toronto (Canada) est une banque de lait d'envergure provinciale qui respecte les lignes directrices établies par la HMBANA. Ainsi, on soumet les donneuses à un questionnaire sur la santé et le mode de vie et à un examen sérologique, et elles reçoivent des conseils sur les procédures adéquates à suivre en ce qui concerne l'expression, la manipulation et l'entreposage du lait maternel. Une fois que les donneuses ont accumulé un certain volume minimal de lait à la maison, le lait est congelé et expédié à la banque par courrier prioritaire express. Nous avons choisi un contenant de lait maternel congelé (environ $150 \mathrm{~mL}$ ) au hasard parmi les envois reçus de 10 donneuses (un contenant par donneuse). Le nombre d'échantillons examiné est cohérent avec des études antérieures sur l'inactivation virale dans les liquides corporels humains, études pour lesquelles il est commun de combiner les échantillons avant l'inoculation avec le virus ${ }^{20-22}$. Nous avons évité de combiner les échantillons dans le cadre de cette étude, étant donné la variabilité connue de la composition du lait maternel. Une fois toutes les données d'identification retirées des contenants de lait, les échantillons ont été transportés à l'état congelé à l'unité combinée de confinement de niveau 3 de l'Université de Toronto, où nous avons réalisé tous nos essais.

Nous avons fait décongeler les échantillons de lait sur de la glace, puis nous les avons homogénéisés et nous avons mélangé 2 aliquotes de $840 \mu \mathrm{L}$ de lait de chaque donneuse avec $160 \mu \mathrm{L}$ de SRAS-CoV-2 SB2 au passage 3 (titre $=6,29 \times 10^{7}, 50 \%$ de la dose infectante de la culture de tissus [DICT ${ }_{50}$ ] par $\mathrm{mL}$ ) afin d'obtenir une concentration de $1 \times 10^{7} \mathrm{DICT}_{50}$ dans chaque $1 \mathrm{~mL}$ de solution à base de lait ${ }^{23}$. Nous avons laissé reposer à la température du laboratoire 1 échantillon infecté de chaque donneuse pendant 30 minutes (lait non pasteurisé). Nous avons pasteurisé le deuxième échantillon infecté de chaque donneuse dans un bain-marie en réchauffant le lait à une température de $62,5^{\circ} \mathrm{C}$ pendant 30 minutes, puis nous avons refroidi les échantillons sur de la glace, conformément aux lignes directrices de la HMBANA et de la EMBA ${ }^{13,14}$. Nous avons ensuite transféré les aliquotes de lait dans des microtubes à centrifuger en plastique rigide, dont la composition était similaire à celle des contenants normalement utilisés pour la pasteurisation à l'aide de la méthode de Holder effectuée à la banque de lait. Sans surprise, le lait maternel s'est révélé cytotoxique pour les cellules Vero E6, et ce, même en l'absence du SRAS-CoV-2, puisqu'il contient des glucides complexes, des lipides et des facteurs immunitaires. Nous avons donc procédé à une dilution 1:100 de tous les échantillons dans le milieu Eagle modifié de Dulbecco (DMEM) sans sérum, le milieu de culture des cellules Vero E6, avant d'effectuer les procédures décrites ci-dessous. Bien que la dilution des échantillons traités entraîne une réduction de 1 log de la sensibilité de détection de virus viable lors du titrage subséquent, le titre élevé du SRAS-CoV-2 utilisé pour inoculer les échantillons de lait compense le facteur de dilution, de sorte qu'une réduction de 6 log de virus viable serait quantifiable.

Comme décrit précédemment, nous avons utilisé des échantillons de lait infectés pour déterminer les charges virales des échantillons dans l'ensemble des conditions expérimentales ${ }^{23,24}$. En résumé, nous avons préparé 6 dilutions en série de facteur 10 de chaque solution de lait contenant le SRAS-CoV-2 (inoculum) et appliqué $50 \mu \mathrm{L}$ de chacune à des cultures monocouches de cellules Vero E6 dans le DMEM (0,2 $\times 10^{6}$ cellules/ $\mathrm{mL}$ ) dans des plaques à fond plat à 96 puits. Nous avons incubé les plaques à $37^{\circ} \mathrm{C}$ et à $5 \%$ de $\mathrm{CO}_{2}$ pendant une heure, en les remuant légèrement aux 15 minutes afin de favoriser une répartition uniforme de l'inoculum dans les puits. Après une heure, nous avons retiré l'inoculum et ajouté aux puits $200 \mu \mathrm{L}$ de DMEM combiné à du sérum bovin fœtal à $2 \%$, puis nous avons laissé le tout incuber pendant 5 jours. Les témoins positifs étaient des échantillons sans lait non dilués composés de $160 \mu \mathrm{L}$ de solution virale dilués dans $840 \mu \mathrm{L}$ de DMEM (SRASCoV-2 uniquement). Les témoins négatifs étaient des échantillons de lait maternel pasteurisés et non pasteurisés sans inoculum viral (infection simulée).

\section{Analyse virologique}

Nous avons évalué les effets cytopathologiques 5 jours après l'infection et nous les avons rapportés en $\mathrm{DICT}_{50} / \mathrm{mL}$. Nous avons calculé les titres viraux à l'aide de la méthode SpearmanKarber ${ }^{25,26}$. Au cinquième jour suivant l'infection, nous avons procédé au passage des surnageants vers de nouvelles cultures monocouches de cellules Vero E6 dans des plaques à fond plat à 
96 puits et rafraîchi les cultures monocouches originales avec $200 \mu \mathrm{L}$ de DMEM combiné à du sérum bovin fœtal à $2 \%$. Pendant 14 jours, nous avons surveillé l'apparition d'effets cytopathologiques non détectables au cours des 5 premiers jours de culture. La limite de détection pour l'essai de DICT ${ }_{50}$ était de $20 \mathrm{DICT}_{50} / \mathrm{mL}$.

\section{Approbation éthique}

Les donneuses de la Rogers Hixon Ontario Human Milk Bank avaient fourni un consentement éclairé par écrit indiquant que leur lait pourrait être utilisé à des fins de contrôle de la qualité ou de recherche. Une approbation d'un comité d'éthique de la recherche sur les humains a été obtenue du système de santé Sinaï et de l'Université de Toronto.

\section{Résultats}

Les effets cytopathologiques étaient identiques au troisième et au cinquième jour suivant l'infection; ils sont présentés dans le tableau 1. Nous n'avons détecté aucune activité cytopathologique dans les échantillons de lait inoculés avec le SRASCoV-2 qui ont été pasteurisés à l'aide de la méthode de Holder (à $62,5{ }^{\circ} \mathrm{C}$ pendant $30 \mathrm{~min}$ ), même après le passage de l'inoculum et la période d'observation subséquente de 14 jours. Il est à noter que dans les échantillons témoins positifs (SRAS-CoV-2 uniquement), ce traitement par la chaleur n'a pas complètement inactivé le virus. Pour ce qui est des échantillons de lait

Tableau 1 : Stabilité du SRAS-CoV-2 dans le lait maternel provenant de donneuses, avec et sans pasteurisation à l'aide de la méthode de Holder $\left(\mathrm{DICT}_{50} / \mathrm{mL}\right)^{\star}$

\begin{tabular}{|c|c|c|}
\hline Échantillons & $\begin{array}{l}\text { Non pasteurisé } \\
\text { (température du } \\
\text { laboratoire pendant } \\
\quad 30 \mathrm{~min} \text { ) }\end{array}$ & $\begin{array}{l}\text { Pasteurisé } \\
\left(62,5^{\circ} \mathrm{C} \text { pendant }\right. \\
30 \mathrm{~min})\end{array}$ \\
\hline A & $2,0 \times 10^{5}$ & Non détecté \\
\hline B & $6,3 \times 10^{4}$ & Non détecté \\
\hline C & $6,3 \times 10^{5}$ & Non détecté \\
\hline D & $6,3 \times 10^{6}$ & Non détecté \\
\hline $\mathrm{E}$ & $6,3 \times 10^{5}$ & Non détecté \\
\hline $\mathrm{F}$ & $2,0 \times 10^{5}$ & Non détecté \\
\hline G & $6,3 \times 10^{5}$ & Non détecté \\
\hline $\mathrm{H}$ & $6,3 \times 10^{5}$ & Non détecté \\
\hline I & $6,3 \times 10^{5}$ & Non détecté \\
\hline J & $6,3 \times 10^{5}$ & Non détecté \\
\hline $\begin{array}{l}\text { SRAS-CoV-2 } \\
\text { uniquement } \\
\text { (témoin positif) }\end{array}$ & $6,3 \times 10^{6}$ & $6,3 \times 10^{3}$ \\
\hline $\begin{array}{l}\text { Infection simulée } \\
\text { (témoin négatif) }\end{array}$ & Non détecté & Non détecté \\
\hline \multicolumn{3}{|c|}{$\begin{array}{l}\text { Remarque : } \text { DICT }_{50}=50 \% \text { de la dose infectante de la culture de tissus, SRAS-CoV-2 = } \\
\text { coronavirus du syndrome respiratoire aigu sévère } 2 \text {. } \\
\text { *Les calculs de la DICT }{ }_{50} / \mathrm{mL} \text { s'appuient sur les dilutions en série répétées des } \\
\text { échantillons indiqués. }\end{array}$} \\
\hline
\end{tabular}

inoculés avec le SRAS-CoV-2 qui n'ont pas été pasteurisés, mais plutôt laissés à la température du laboratoire pendant $30 \mathrm{~min}$ utes, nous avons enregistré une réduction d'environ 1 log par rapport au virus ajouté seul dans le DMEM; nous avons toutefois observé une variabilité entre les donneuses (d'une réduction de 2 log à aucune réduction). La moyenne et l'écart-type étaient de $1,05 \times 10^{6} \pm 1,86 \times 10^{6}$ pour la $\mathrm{DICT}_{50} / \mathrm{mL}$.

\section{Interprétation}

Très peu d'échantillons de lait de femmes atteintes de la COVID-19 ont été testés pour la détection du SRAS-CoV-2. Parmi les quelques cas décrits dans la documentation, il y a aujourd'hui au moins 3 cas répertoriés où des acides nucléiques de SRAS-CoV-2 ont été détectés dans le lait maternel; cependant, la viabilité du virus dans ces échantillons n'a pas été mesurée. L'Organisation mondiale de la Santé recommande que les nourrissons ayant un très faible poids à la naissance reçoivent du lait maternel provenant de donneuses lorsque le volume produit par la mère est insuffisant ${ }^{27}$. Le réseau de banques de lait maternel croît rapidement aux quatre coins du monde : il y a aujourd'hui plus de 650 banques de lait qui emploient la méthode de Holder pour assurer la sécurité des dons ${ }^{28}$.

Bien que l'on pense que cette technique puisse entraîner une inactivation du SRAS-CoV-2, il est important de confirmer cette hypothèse dans une matrice de lait maternel, pour la sécurité du personnel des banques de lait, des travailleurs de la santé et des receveurs de dons de lait maternel. Dans la présente étude, la pasteurisation du lait maternel inoculé avec le SRAS-CoV-2 à l'aide de la méthode de Holder (à $62,5^{\circ} \mathrm{C}$ pendant $30 \mathrm{~min}$ ) a occasionné une inactivation virale complète, mesurée par la $\mathrm{DICT}_{50} /$ $\mathrm{mL}$. La charge virale élevée utilisée pour infecter les échantillons dans le cadre de ces expériences nous a permis de confirmer une réduction de l'ordre de $10^{6}$. Les effets de la pasteurisation sur les coronavirus dans une matrice de lait maternel n'avaient jamais été décrits dans la documentation ${ }^{29}$.

Nos résultats vont dans le même sens que les données montrant une inactivation du coronavirus dans d'autres matrices, dont les milieux de culture et le plasma, à l'aide de différents protocoles de pasteurisation. Il a également été établi que le virus causant le syndrome respiratoire aigu sévère, le SRAS-CoV, pouvait être complètement inactivé lorsqu'exposé à des températures aussi basses que $56^{\circ} \mathrm{C}$ pendant 20 minutes, de même qu'à des températures plus élevées, par exemple $70^{\circ} \mathrm{C}$ pendant cinq minutes ${ }^{30-33}$. Par ailleurs, on a montré que le virus causant le syndrome respiratoire du Moyen-Orient pouvait être inactivé lorsqu'exposé à une température de $56{ }^{\circ} \mathrm{C}$ durant 60 minutes ${ }^{34,35}$. Un récent article de Chin et de ses collègues a conclu que le SRAS-CoV-2 pouvait être complètement inactivé lorsqu'exposé à une température de $56{ }^{\circ} \mathrm{C}$ pendant 30 minutes ou de $70{ }^{\circ} \mathrm{C}$ pendant 5 minutes dans un milieu de transport viral ${ }^{19}$. Dans le cadre de la présente étude, nous n'avons pas observé d'inactivation complète du SRAS-CoV-2 dans un milieu qui ne contenait pas de lait maternel (témoin positif) après une pasteurisation à $62,5^{\circ} \mathrm{C}$ pendant 30 minutes, ce qui ne correspond 
pas aux données rapportées par Chin et ses collègues. À notre avis, ces résultats indiquent que la matrice biologique du virus doit être prise en compte lors de l'évaluation des conditions d'une inactivation efficace.

Fait intéressant, nous avons noté une réduction des effets cytopathologiques du SRAS-CoV-2 dans les échantillons de lait qui n'ont pas été soumis à la chaleur, mais plutôt laissés à la température du laboratoire pendant 30 minutes. Il est fort probable que cet effet soit dû aux multiples composantes immunitaires du lait maternel, dont les anticorps IgA sécrétoires, la lactoferrine, la lactadhérine, les mucines des globules gras du lait et les oligosaccharides, qui présentent une activité antivirale significative ${ }^{1,2}$. D'ailleurs, Hamilton Spence et ses collègues ont constaté le même phénomène dans des échantillons de lait maternel inoculés avec le virus Ebola et conservés à la température du laboratoire pendant 30 minutes $^{36}$.

\section{Limites de l'étude}

Nous n'avons étudié que 10 échantillons de lait. Notre capacité d'analyse d'un plus grand nombre d'échantillons était limitée en raison de la complexité des mesures de sécurité à prendre avec le virus SRAS-CoV-2; néanmoins, la taille de notre échantillon est plus importante que celle utilisée dans des études semblables s'intéressant à d'autres virus ${ }^{20-22}$.

\section{Conclusion}

La pasteurisation de lait maternel à l'aide de la méthode de Holder (à $62,5^{\circ} \mathrm{C}$ pendant $30 \mathrm{~min}$ ) permet d'inactiver le SRASCoV-2. Si une femme atteinte de la COVID-19 faisait un don de lait contenant le SRAS-CoV-2, que ce soit en raison d'une transmission par la glande mammaire ou d'une contamination par des gouttelettes respiratoires, la peau, un tire-lait ou un contenant de lait, cette méthode de pasteurisation rendrait le lait sûr à la consommation par le nourrisson. De plus, le lait maternel décongelé (préalablement congelé) semble présenter une activité antivirale assez importante pour réduire partiellement l'infectivité du SRAS-CoV-2 qu'il contient.

\section{Références}

1. Goldman A, Goldblum R. Chapter 9 - Defense agents in milk: A. Defense agents in human milk. In: Jensen R, editor. Handbook of Milk Composition: $A$ Volume in Food Science and Technology. San Diego (CA): Academic Press; 1995:727-45

2. Goldman AS. Future research in the immune system of human milk. J Pediatr 2019;206:274-9.

3. Hurley WL, Theil PK. Perspectives on immunoglobulins in colostrum and milk. Nutrients 2011;3:442-74.

4. Maertens K, De Schutter S, Braeckman T, et al. Breastfeeding after maternal immunisation during pregnancy: providing immunological protection to the newborn: a review. Vaccine 2014;32:1786-92.

5. Victora CG, Bahl R, Barros AJ, et al.; Lancet Breastfeeding Series Group. Breastfeeding in the 21st century: epidemiology, mechanisms, and lifelong effect. Lancet 2016;387:475-90.

6. O'Connor DL, Gibbins S, Kiss A, et al.; GTA DoMINO Feeding Group. Effect of supplemental donor human milk compared with preterm formula on neurodevelopment of very low-birth-weight infants at 18 months: a randomized clinical trial. JAMA 2016;316:1897-905.
7. O'Connor DL, Jacobs J, Hall R, et al. Growth and development of premature infants fed predominantly human milk, predominantly premature infant formula, or a combination of human milk and premature formula. J Pediatr Gastroenterol Nutr 2003;37:437-46.

8. Patel AL, Johnson TJ, Engstrom JL, et al. Impact of early human milk on sepsis and health-care costs in very low birth weight infants. J Perinatol 2013;33: 514-9.

9. Quigley M, Embleton ND, McGuire W. Formula versus donor breast milk for feeding preterm or low birth weight infants. Cochrane Database Syst Rev 2018;6:CD002971.

10. Kim J, Unger S. Human milk banking. Paediatr Child Health 2010;15:595-602.

11. Vogel L. Milk sharing: Boon or biohazard? CMAJ 2011;183:E155-6.

12. Lawrence RM, Lawrence RA. Breast milk and infection. Clin Perinatol 2004; 31:501-28.

13. HMBANA. Guidelines for the establishment and operation of a donor human milk bank. 10th ed. Fort Worth (TX): Human Milk Banking Association of North America (HMBANA); 2018.

14. Moro GE, Billeaud C, Rachel B, et al. Processing of donor human milk: update and recommendations from the European Milk Bank Association (EMBA). Front Pediatr 2019;7:49.

15. Groß R, Conzelmann C, Müller J, et al. Detection of SARS-CoV-2 in human breastmilk. Lancet 2020;395:1757-8.

16. Kirtsman M, Diambomba Y, Poutanen SM, et al. Probable congenital SARS-CoV-2 infection in a neonate born to a woman with active SARS-CoV-2 infection. CMAJ 2020;192:E647-50.

17. Lackey KA, Pace RM, Williams JE, et al. SARS-CoV-2 and human milk: What is the evidence? Matern Child Nutr 2020 May 30 [Cyberpublication avant impression]. doi: 10.1111/mcn.13032.

18. Wu Y, Liu C, Dong L, et al. Viral shedding of COVID-19 in pregnant women. SSRN. doi: 10.2139/ssrn.3562059.

19. Chin AW, Chu J, Perera M, et al. Stability of SARS-CoV-2 in different environmental conditions. Lancet Microbe 2020;1:e10. doi: 10.1016/S2666-5247(20) 30003-3.

20. Eickmann M, Gravemann U, Handke W, et al. Inactivation of Ebola virus and Middle East respiratory syndrome coronavirus in platelet concentrates and plasma by ultraviolet $\mathrm{C}$ light and methylene blue plus visible light, respectively. Transfusion 2018;58:2202-7.

21. Hashem AM, Hassan AM, Tolah AM, et al. Amotosalen and ultraviolet A light efficiently inactivate MERS-coronavirus in human platelet concentrates. Transfus Med 2019;29:434-41.

22. Laughhunn A, Huang Y-SS, Vanlandingham DL, et al. Inactivation of chikungunya virus in blood components treated with amotosalen/ultraviolet $\mathrm{A}$ light or amustaline/glutathione. Transfusion 2018;58:748-57.

23. Banerjee A, Nasir JA, Budylowski P, et al. Isolation, sequence, infectivity, and replication kinetics of severe acute respiratory syndrome coronavirus 2. Emerg Infect Dis 2020;26 [Cyberpublication avant impression]. doi: 10.3201/eid2609.201495.

24. Caly L, Druce J, Roberts J, et al. Isolation and rapid sharing of the 2019 novel coronavirus (SARS-CoV-2) from the first patient diagnosed with COVID-19 in Australia. Med J Aust 2020;212:459-62.

25. Hamilton M, Russo R, Thurston R. Trimmed Spearman-Karber method for estimating median lethal concentrations in toxicity bioassays. Environ Sci Technol 1977;11:714-9. doi: 10.1021/es60130a004.

26. Spearman C. The method of 'right and wrong cases' ('constant stimuli') without Gauss's formulae. Br J Psychol 1904-1920 1908;2:227-42. doi: 10.1111/j.2044-8295 .1908.tb00176.x

27. Donor human milk for low-birth-weight infants. Geneva: World Health Organization; updated 2019. Available: www.who.int/elena/titles/donormilk_ infants/en/ (accessed 2020 June 1).

28. Haiden N, Ziegler EE. Human milk banking. Ann Nutr Metab 2016;69(Suppl 2): 8-15.

29. Pitino MA, O'Connor DL, McGeer AJ, et al. The impact of thermal pasteurization on viral load in human milk and other matrices: a rapid review. medRxiv 2020 May 27. doi: 10.1101/2020.05.23.20111369. 
30. Darnell ME, Subbarao K, Feinstone SM, et al. Inactivation of the coronavirus that induces severe acute respiratory syndrome, SARS-CoV. J Virol Methods 2004;121:85-91.

31. Duan S-M, Zhao X-S, Wen R-F, et al.; SARS Research Team. Stability of SARS coronavirus in human specimens and environment and its sensitivity to heating and UV irradiation. Biomed Environ Sci 2003;16:246-55.

32. Kariwa H, Fujii N, Takashima I. Inactivation of SARS coronavirus by means of povidone-iodine, physical conditions and chemical reagents. Dermatology 2006;212(Suppl 1):119-23.
33. Yunoki M, Urayama T, Yamamoto I, et al. Heat sensitivity of a SARS-associated coronavirus introduced into plasma products. Vox Sang 2004;87:302-3.

34. Leclercq I, Batéjat $C$, Burguière AM, et al. Heat inactivation of the Middle East respiratory syndrome coronavirus. Influenza Other Respir Viruses 2014;8:585-6.

35. van Doremalen N, Bushmaker T, Karesh WB, et al. Stability of Middle East respiratory syndrome coronavirus in milk. Emerg Infect Dis 2014;20:1263-4.

36. Hamilton Spence E, Huff M, Shattuck K, et al. Ebola virus and Marburg virus in human milk are inactivated by Holder pasteurization. J Hum Lact 2017;33:351-4.
Intérêts concurrents : Deborah O'Connor est présidente du conseil consultatif (non rémunérée) et Sharon Unger est directrice médicale (rémunérée) de la Rogers Hixon Ontario Human Milk Bank. Aucun autre intérêt concurrent déclaré.

Cet article a été révisé par des pairs.

Affiliations : Rogers Hixon Ontario Human Milk Bank (Unger, O'Connor); Département de pédiatrie, Sinai Health (Unger); unité combinée de confinement de niveau 3 (Christie-Holmes, Guvenc, Budylowski, Gray-Owen), Départements de génétique moléculaire (Guvenc, GrayOwen), de médecine de laboratoire et pathobiologie (Mubareka) et de nutrition (O'Connor), et Institut des sciences médicales (Budylowski), Université de Toronto; Institut de recherche Sunnybrook (Mubareka), Toronto (Ontario)
Collaborateurs : Tous les auteurs ont contribué à l'élaboration et à la conception des travaux ainsi qu'à l'acquisition, à l'analyse et à l'interprétation des données. Sharon Unger, Natasha Christie-Holmes et Deborah O'Connor ont rédigé le manuscrit. Tous les auteurs ont révisé de façon critique le contenu intellectuel important du manuscrit; ils ont donné leur approbation finale pour la version destinée à être publiée et assument l'entière responsabilité de tous les aspects du travail. Sharon Unger et Natasha Christie-Holmes sont toutes deux premières auteures.

Financement : Ces travaux de recherche ont été financés par les Instituts de recherche en santé du Canada (FDN n ${ }^{\circ}$ 143233). Un appui indirect a également été obtenu de
I'Université de Toronto et de la Temerty Foundation dans le but de soutenir une capacité et des activités accrues de l'unité combinée de niveau de confinement 3 de Toronto durant la pandémie de COVID-19. Le soutien n'a pas influencé le modèle ni le déroulement des travaux, l'interprétation des données ou la rédaction du présent manuscrit.

Partage des données : Les demandes pour obtenir les données originales doivent être soumises à la $\mathrm{D}^{\mathrm{re}}$ Sharon Unger, à l'adresse : sharon.unger@sinaihealth.ca.

Accepté : 30 juin 2020

Correspondance : Sharon Unger, sharon.unger@sinaihealth.ca 